\title{
Enzymatic Vitreolysis with Ocriplasmin for Vitreomacular Traction and Macular Holes
}

\author{
Peter Stalmans, M.D., Ph.D., Matthew S. Benz, M.D., Arnd Gandorfer, M.D., \\ Anselm Kampik, M.D., Aniz Girach, M.D., Stephen Pakola, M.D., \\ and Julia A. Haller, M.D., for the MIVI-TRUST Study Group*
}

ABSTRACT

From the Department of Ophthalmology, Universitaire Ziekenhuizen Leuven, Leuven, Belgium (P.S.); Retina Consultants of Houston, Houston (M.S.B.); the Department of Ophthalmology, Klinikum der Universität Munich, Munich (A. Gandorfer, A.K.), and Medizinisches Versorgungszentrum, Memmingen (A. Gandorfer) - both in Germany; ThromboGenics, Heverlee, Belgium (A. Girach, S.P.); and Wills Eye Institute, Philadelphia (J.A.H.). Address reprint requests to Dr. Haller at the Wills Eye Institute, $840 \mathrm{Wal}$ nut St., Suite 1510, Philadelphia, PA 19107, or at jhaller@willseye.org.

*Investigators participating in the Microplasmin for Intravitreous Injection Traction Release without Surgical Treatment (MIVI-TRUST) clinical program are listed in the Supplementary Appendix, available at NEJM.org.

This article was updated on November 22, 2012, at NEJM.org.

N EnglJ Med 2012;367:606-15. DOI: 10.1056/NEJMoall10823

Copyright (c) 2012 Massachusetts Medical Society.

\section{BACKGROUND}

Vitreomacular adhesion can lead to pathologic traction and macular hole. The standard treatment for severe, symptomatic vitreomacular adhesion is vitrectomy. Ocriplasmin is a recombinant protease with activity against fibronectin and laminin, components of the vitreoretinal interface.

\section{METHODS}

We conducted two multicenter, randomized, double-blind, phase 3 clinical trials to compare a single intravitreal injection of ocriplasmin $(125 \mu \mathrm{g})$ with a placebo injection in patients with symptomatic vitreomacular adhesion. The primary end point was resolution of vitreomacular adhesion at day 28. Secondary end points were total posterior vitreous detachment and nonsurgical closure of a macular hole at 28 days, avoidance of vitrectomy, and change in best-corrected visual acuity.

\section{RESULTS}

Overall, 652 eyes were treated: 464 with ocriplasmin and 188 with placebo. Vitreomacular adhesion resolved in $26.5 \%$ of ocriplasmin-injected eyes and in $10.1 \%$ of placebo-injected eyes $(\mathrm{P}<0.001)$. Total posterior vitreous detachment was more prevalent among the eyes treated with ocriplasmin than among those injected with placebo $(13.4 \%$ vs. $3.7 \%, \mathrm{P}<0.001)$. Nonsurgical closure of macular holes was achieved in $40.6 \%$ of ocriplasmin-injected eyes, as compared with $10.6 \%$ of placeboinjected eyes $(\mathrm{P}<0.001)$. The best-corrected visual acuity was more likely to improve by a gain of at least three lines on the eye chart with ocriplasmin than with placebo. Ocular adverse events (e.g., vitreous floaters, photopsia, or injection-related eye pain - all self-reported - or conjunctival hemorrhage) occurred in $68.4 \%$ of ocriplasmininjected eyes and in $53.5 \%$ of placebo-injected eyes $(\mathrm{P}<0.001)$, and the incidence of serious ocular adverse events was similar in the two groups $(\mathrm{P}=0.26)$.

\section{CONCLUSIONS}

Intravitreal injection of the vitreolytic agent ocriplasmin resolved vitreomacular traction and closed macular holes in significantly more patients than did injection of placebo and was associated with a higher incidence of ocular adverse events, which were mainly transient. (Funded by ThromboGenics; ClinicalTrials.gov numbers, NCT00781859 and NCT00798317.) 
T HE HUMAN VITREOUS BODY IS BOUNDED posteriorly by the retina and is variably adherent to it. Collagen fibrils forming the posterior vitreous cortex are firmly attached at the macula, the central part of the retina where visual acuity is best, and are connected to its internal limiting membrane by means of a biochemical glue composed of proteoglycans, including laminin and fibrinectin. ${ }^{1-4}$ With aging, the gel-like vitreous progressively liquefies and vitreoretinal adhesions weaken, leading to separation of the vitreous from the retina, or posterior vitreous detachment. ${ }^{5-7}$

Vitreomacular adhesion is observed after partial posterior vitreous detachment, when a portion of the posterior vitreous remains attached to the macula. When traction increases in response to anteroposterior forces, tangential forces, or both, the adhesion may cause vitreomacular traction and become symptomatic. Symptoms typically include metamorphopsia and blurring of visual acuity with central visual-field defects. Vitreomacular traction can lead to macular distortion and edema and to the formation of macular holes. ${ }^{8}$ It has been suggested that vitreomacular adhesion may play a role in the progression of diabetic retinopathy and agerelated macular degeneration.,9-12 Vitrectomy is the only treatment for vitreomacular traction and macular holes, and because it poses certain risks (infection, retinal detachment, hemorrhage, and cataract), it is usually withheld until loss of vision has become clinically significant.

Vitreolysis involving an enzyme that has activity against the molecular substrates responsible for vitreomacular adhesion is a potential nonsurgical, biologic approach to the treatment of this disorder. Substances directed against biochemical components of the vitreomacular interface, such as chondroitinase, dispase, and hyaluronidase, have been tested but were abandoned because of insufficient clinical efficacy, complications, or both. ${ }^{13-15}$ Plasmin and a truncated form of plasmin, ocriplasmin (formerly microplasmin), have been shown to be effective in ex vivo studies of vitreolysis in animals and humans. ${ }^{16-18}$ Such an approach to symptomatic vitreomacular adhesion could allow nonsurgical intervention, treatment at an earlier stage of the disease, or both, which would probably result in better outcomes. . $7,13-15,19$

Ocriplasmin is a truncated form of the human serine protease plasmin and has proteolytic activity against fibronectin and laminin, two major components of the vitreoretinal interface. Results of preclinical and clinical studies have suggested that ocriplasmin can induce vitreous liquefaction and separation from the retina. ${ }^{17,18,20}$ Phase 2 studies have shown that intravitreal injection of a single $125-\mu \mathrm{g}$ dose of ocriplasmin or up to three injections of $125 \mu \mathrm{g}$ each given monthly can induce the resolution of vitreomacular traction and closure of macular holes without causing serious adverse events. ${ }^{1,21}$

\section{METHODS}

\section{STUDY DESIGN AND OVERSIGHT}

We performed two multicenter, randomized, double-blind, placebo-controlled, phase 3 studies (TG-MV-006 and TG-MV-007, hereafter called study 006 and study 007) to test the efficacy and safety of a single intravitreal injection of ocriplasmin. Patients were randomly assigned to intravitreal injection of either ocriplasmin or placebo. Owing to a specific recommendation by the Food and Drug Administration, the ratio of randomized assignments to ocriplasmin and placebo in study 006 was changed to $2: 1$, and the randomization ratio in study 007 was 3:1 from the outset. Otherwise, the study protocols were identical. In study 006, enrollment began on December 23, 2008, and was completed on September 4, 2009. In study 007, enrollment began on December 22, 2008, and was completed on September 17, 2009. The studies were approved by the institutional review board or independent ethics committee at each participating site, and we obtained written informed consent from all patients before they were enrolled.

Both trials were designed, coordinated, and sponsored by ThromboGenics. The data were gathered independently by the Microplasmin for Intravitreous Injection - Traction Release without Surgical Treatment (MIVI-TRUST) study groups and were analyzed by contract research organizations paid by ThromboGenics. The authors wrote the manuscript and made the decision, in consultation with ThromboGenics, to submit it for publication. The authors attest that the studies were performed in accordance with the protocols, including the statistical analysis plans, available with the full text of this article at NEJM.org. The authors vouch for the accuracy and completeness of the reported results and for the fidelity of this report to the study protocols. 
INCLUSION AND EXCLUSION CRITERIA

Patients were eligible for the study if they were at least 18 years of age and had focal vitreomacular adhesion, defined as vitreous adhesion to the macula within a 6-mm central retinal field surrounded by elevation of the posterior vitreous cortex, as seen on optical coherence tomography (OCT), and a best-corrected visual acuity of 20/25 or less in the study eye and 20/800 or more in the nonstudy eye, according to the Early Treatment Diabetic Retinopathy Study (ETDRS) acuity chart. Patients were excluded if they had proliferative diabetic retinopathy, neovascular age-related macular degeneration, retinal vascular occlusion, aphakia, high myopia (more than -8 diopters), uncontrolled glaucoma, a macular hole greater than $400 \mu \mathrm{m}$ in diameter, vitreous opacification, lenticular or zonular instability, or a history of retinal detachment in either eye. Additional exclusion criteria were prior vitrectomy, prior laser photocoagulation of the macula, and treatment with ocular surgery, intravitreal injection, or retinal laser photocoagulation in the previous 3 months. The presence of an epiretinal membrane was not a criterion for exclusion.

\section{TREATMENT}

Patients randomly assigned to the ocriplasmin group received an intravitreal injection of ocriplasmin (125 $\mu \mathrm{g}$ in a $0.10-\mathrm{ml}$ volume) drawn from a vial containing ocriplasmin into which $0.75 \mathrm{ml}$ of commercial saline had been injected (1875 $\mu \mathrm{g}$ of ocriplasmin in a $0.75-\mathrm{ml}$ drug vehicle). Patients randomly assigned to placebo received an intravitreal injection of $0.10 \mathrm{ml}$ of the identical drug vehicle diluted with saline, the method used being the same as that used to prepare ocriplasmin. Assessments were made at baseline, on the day of injection, and on days 7, 14, 28, 90, and 180 after the injection. Investigators could proceed to recommend vitrectomy at any time if the underlying condition deteriorated, if the best-corrected visual acuity in the study eye worsened by more than two lines on the eye chart, or if the underlying condition had not improved within 4 weeks after the injection.

\section{ASSESSMENTS}

Assessments included a complete ophthalmic examination (measurement of best-corrected visual acuity, manifest refraction, and intraocular pressure; slit-lamp examination; and ophthalmoscopy with pupillary dilation), B-scan ultrasonography to evaluate the status of the posterior vitreous cortex, OCT to document the status of vitreomacular adhesion and the presence or absence of a macular hole, fundus photography, and fluorescein angiography. Patients completed the selfadministered National Eye Institute Visual Functioning Questionnaire-25 (VFQ-25) (August 2000 version, translated into the native languages of the patients), ${ }^{22,23}$ which assesses visual function and general health on a scale from 0 to 100 , with a score of 100 indicating optimal function and health.

Best-corrected visual acuity was reported as the number of letters correctly read by the patient on an ETDRS chart at $4 \mathrm{~m}$. OCT measurements obtained with the use of the time-domain Stratus device (Zeiss) were mandatory. In addition, spectral-domain OCT images were obtained when the devices required for such images were available. Trained readers at a central reading center (Duke University OCT Reading Center, Durham, NC) who were unaware of the group assignments evaluated the OCT images. All ultrasonographic studies were standardized and performed by certified technicians who underwent special training for the study. Staging of posterior vitreous detachment was based on dynamic ultrasonographic evaluation and performed by an investigator who was unaware of the group assignments (see Table S1 in the Supplementary Appendix, available at NEJM.org). ${ }^{24,25}$ Cataracts were graded on the basis of standardized photographs according to the methods used in the Age-Related Eye Disease Study 2008 system. ${ }^{26}$

\section{STUDY END POINTS}

The primary end point was the percentage of eyes with nonsurgical resolution of vitreomacular adhesion at day 28 , as determined by the OCT evaluation obtained from the central reading center. The main secondary end point was the percentage of eyes with total posterior vitreous detachment at day 28 , as determined by the investigator from standardized B-scan ultrasonograms. Predetermined secondary end points included the need for vitrectomy, closure of a macular hole, a gain of three or more lines in the assessment of bestcorrected visual acuity without vitrectomy, and change from baseline in best-corrected visual acuity and VFQ-25 score at 6 months. ${ }^{23,27}$ 


\section{STATISTICAL ANALYSIS}

Assuming that the rate of the primary end point was $27.5 \%$ in the ocriplasmin group and $10.0 \%$ in the placebo group, we determined that a sample of 320 patients would provide more than $90 \%$ power to detect a significant difference at a two-sided alpha level of 0.05 . For all planned and exploratory analyses, statistical significance was set at an alpha level of 0.05 . All statistical tests and confidence intervals were two-sided. Statistical analysis of the primary and secondary end points was performed on the combined data from both trials (i.e., all patients who underwent randomization, according to the intention-to-treat principle) by carrying the last observation forward as a conservative approach to impute any missing data.

For binary end points, group comparisons in the individual studies were carried out with the use of Fisher's exact test. Treatment effects are expressed as odds ratios and corresponding exact $95 \%$ confidence intervals. Homogeneity of the odds ratios across the two studies was assessed by means of a Breslow-Day test. Results were combined and analyzed by means of a CochranMantel-Haenszel test and were stratified according to the study. We derived common odds ratios, with their associated exact 95\% confidence intervals, with the use of a logistic-regression model that included factors for study group and for study.

We compared changes in best-corrected visual acuity and VFQ-25 scores between the two study groups with the use of an analysis-of-variance model, adjusted with a factor for baseline bestcorrected visual acuity and VFQ-25 score. For the combined analysis, a mixed-model analysis of variance was used, including a fixed effect for study group and a random effect for study. For the safety analyses, patients were evaluated according to the study medication they actually received.

\section{RESULTS}

\section{PATIENTS}

In total, 652 patients were enrolled; 464 patients were randomly assigned to receive an intravitreal ocriplasmin injection and 188 to receive a placebo injection. The study groups had similar demographic and baseline disease characteristics, with two exceptions: pseudophakia was more common in the ocriplasmin group than in the placebo group
(37.1\% vs. $28.2 \%$ ), and there were more women in the ocriplasmin group than in the placebo group (67.7\% vs. 61.2\%) (Table S2 in the Supplementary Appendix).

\section{END POINTS}

The primary end point, the proportion of patients with nonsurgical resolution of vitreomacular adhesion on OCT at day 28 , was significantly higher with ocriplasmin than with placebo in each of the two studies $(\mathrm{P}=0.003$ in study 006 and $\mathrm{P}<0.001$ in study 007). We found no significant evidence for heterogeneity of treatment effects between the two studies $(\mathrm{P}=0.23)$. For the combined studies, the odds ratio for intervention was 3.28 on the primary end point ( $95 \%$ confidence interval [CI], 1.93 to $5.84 ; \mathrm{P}<0.001$ ) (Table 1 ). Table 1 shows the treatment effects on the primary and secondary end points in the two individual studies and for the combined data. The difference between the ocriplasmin and placebo groups was significant for all prespecified end points in the combined analyses. Overall, $26.5 \%$ of the patients in the ocriplasmin group reached the primary end point (resolution of vitreomacular adhesion), as compared with $10.1 \%$ of those in the placebo group (Fig. 1A). The between-group difference in the percentage of eyes with resolution of vitreomacular adhesion reached significance at day 7 , the day of the first study visit after the injection (odds ratio for nonsurgical resolution with ocriplasmin, 5.20; 95\% CI, 2.53 to 12.07 ; $\mathrm{P}<0.001$ ), and the percentage remained higher with ocriplasmin at all subsequent visits through month 6 (Fig. 1A).

The magnitude of the effect of ocriplasmin on the primary end point varied according to lens status. Among phakic eyes, $34.2 \%$ in the ocriplasmin group versus $12.6 \%$ in the placebo group had resolution of vitreomacular adhesion (odds ratio, 3.75; 95\% CI, 2.09 to 7.07; $\mathrm{P}<0.001$ ); among eyes with pseudophakia, $13.4 \%$ in the ocriplasmin group versus $3.8 \%$ in the placebo group had resolution of vitreomacular adhesion (odds ratio, 3.96; 95\% CI, 0.92 to 35.89; $\mathrm{P}=0.051$ ). Resolution of vitreomacular adhesion was achieved in $18.7 \%$ of men who received ocriplasmin versus $5.5 \%$ of those who received placebo (odds ratio, 3.83; 95\% CI, 1.25 to $15.75 ; \mathrm{P}=0.01$ ) and in $30.3 \%$ of women who received ocriplasmin versus $13.0 \%$ of those who received placebo (odds ratio, 2.94; 95\% CI, 1.60 to $5.76 ; \mathrm{P}<0.001$ ). 


\begin{tabular}{|c|c|c|c|c|}
\hline Variable & Placebo & Ocriplasmin & Odds Ratio $(95 \% \mathrm{CI}) *$ & P Value \\
\hline \multicolumn{5}{|c|}{ no. of events/total no. of patients (\%) } \\
\hline \multicolumn{5}{|c|}{ Resolution of vitreomacular adhesion at day 28 (homogeneity of treatment effect, $\mathrm{P}=0.23$ ) } \\
\hline Study 006 & $14 / 107(13.1)$ & $61 / 219(27.9)$ & $2.56(1.32-5.24)$ & 0.003 \\
\hline Study 007 & $5 / 81(6.2)$ & $62 / 245(25.3)$ & $5.13(1.97-17.00)$ & $<0.001$ \\
\hline Combined data & $19 / 188(10.1)$ & $123 / 464(26.5)$ & $3.28(1.93-5.84)$ & $<0.001$ \\
\hline \multicolumn{5}{|c|}{ Total posterior vitreous detachment at day 28 (homogeneity of treatment effect, $\mathrm{P}=0.07$ ) } \\
\hline Study 006 & $7 / 107(6.5)$ & $36 / 219(16.4)$ & $2.80(1.17-7.74)$ & 0.01 \\
\hline Study 007 & $0 / 81(0)$ & $26 / 245(10.6)$ & $13.55(2.35-\infty)$ & $<0.001$ \\
\hline Combined data & $7 / 188(3.7)$ & $62 / 464(13.4)$ & $4.27(1.89-11.32)$ & $<0.001$ \\
\hline \multicolumn{5}{|c|}{ Closure of macular hole at day 28 (homogeneity of treatment effect, $\mathrm{P}=0.74$ ) } \\
\hline Study 006 & $4 / 32(12.5)$ & $25 / 57(43.9)$ & $5.37(1.58-23.84)$ & 0.002 \\
\hline Study 007 & $1 / 15(6.7)$ & $18 / 49(36.7)$ & $7.94(1.04-362.47)$ & 0.03 \\
\hline Combined data & $5 / 47(10.6)$ & $43 / 106(40.6)$ & $5.94(2.09-21.01)$ & $<0.001$ \\
\hline \multicolumn{5}{|c|}{ Improvement in visual acuity $\geq 3$ lines at month 6 (homogeneity of treatment effect, $P=0.28$ ) } \\
\hline Study 006 & $9 / 107(8.4)$ & $28 / 219(12.8)$ & $1.59(0.70-4.00)$ & 0.27 \\
\hline Study 007 & $3 / 81(3.7)$ & $29 / 245(11.8)$ & $3.48(1.03-18.35)$ & 0.03 \\
\hline Combined data & $12 / 188(6.4)$ & $57 / 464(12.3)$ & $2.09(1.08-4.41)$ & 0.02 \\
\hline \multicolumn{5}{|c|}{ Vitrectomy at month 6 (homogeneity of treatment effect, $P=0.83$ ) } \\
\hline Study 006 & $31 / 107(29.0)$ & $45 / 219(20.5)$ & $0.64(0.36-1.12)$ & 0.10 \\
\hline Study 007 & $19 / 81(23.5)$ & $37 / 245(15.1)$ & $0.58(0.30-1.15)$ & 0.09 \\
\hline Combined data & $50 / 188(26.6)$ & $82 / 464(17.7)$ & $0.61(0.40-0.94)$ & 0.02 \\
\hline \multicolumn{5}{|c|}{ Any ocular adverse event (homogeneity of treatment effect, $\mathrm{P}=0.73$ ) } \\
\hline Study 006 & $62 / 106(58.5)$ & $159 / 220(72.3)$ & $1.85(1.10-3.09)$ & 0.02 \\
\hline Study 007 & $38 / 81(46.9)$ & $159 / 245(64.9)$ & $2.09(1.22-3.60)$ & 0.006 \\
\hline Combined data & $100 / 187(53.5)$ & $318 / 465(68.4)$ & $1.96(1.36-2.82)$ & $<0.001$ \\
\hline \multicolumn{5}{|c|}{ Any ocular serious adverse event (homogeneity of treatment effect, $\mathrm{P}=0.34$ ) } \\
\hline Study 006 & $11 / 106(10.4)$ & $21 / 220(9.5)$ & $0.91(0.40-2.18)$ & 0.84 \\
\hline Study 007 & $9 / 81$ (11.1) & $15 / 245(6.1)$ & $0.52(0.20-1.42)$ & 0.15 \\
\hline Combined data & $20 / 187(10.7)$ & $36 / 465$ (7.7) & $0.72(0.39-1.36)$ & 0.26 \\
\hline
\end{tabular}

* Cl denotes confidence interval.

A prespecified subgroup analysis based on the presence or absence of an epiretinal membrane showed that among patients without an epiretinal membrane, $37.4 \%$ in the ocriplasmin group had nonsurgical resolution of vitreomacular adhesion, as compared with $14.3 \%$ in the placebo group (odds ratio, 3.79; 95\% CI, 2.09 to 7.22; $\mathrm{P}<0.001$ ) (Fig. 1B). Among patients with an epiretinal membrane, resolution of vitreomacular adhesion occurred in $8.7 \%$ of those in the ocriplasmin group as compared with $1.5 \%$ of those in the placebo group (odds ratio, 6.20; $95 \% \mathrm{CI}, 0.93$ to 265.068; $\mathrm{P}=0.046$ ).

Total posterior vitreous detachment at day 28 was noted on ultrasonography in $13.4 \%$ of eyes injected with ocriplasmin, as compared with $3.7 \%$ of eyes injected with placebo (odds ratio, 4.27; 95\% CI, 1.89 to 11.32; P<0.001) (Fig. 2A). Nonsurgical closure of a macular hole by day 28 was achieved in $40.6 \%$ of eyes injected with ocriplasmin, as compared with $10.6 \%$ of eyes injected with placebo (odds ratio, 5.94; 95\% CI, 2.09 to 21.01; P $<0.001$ ) (Fig. 2B, and Fig. S1 in the Supplementary Appendix), and the percentage remained higher with ocriplasmin at the end of the study (40.6\%, vs. $17.0 \%$ with placebo; odds ratio, 3.45 ; $95 \%$ CI, 1.40 to $9.49 ; \mathrm{P}=0.004)$.

During the studies, some patients underwent vitrectomy, in most instances to treat persistent vitreomacular adhesion. At 6 months, fewer pa- 
tients in the ocriplasmin group than in the placebo group had undergone vitrectomy $(17.7 \%$ vs. $26.6 \%$; odds ratio, $0.61 ; 95 \% \mathrm{CI}, 0.40$ to $0.94 ; \mathrm{P}=0.02$ ).

In the total population of patients, an improvement in best-corrected visual acuity of three or more lines on the eye chart was achieved in $12.3 \%$ of eyes injected with ocriplasmin, as compared with $6.4 \%$ of eyes injected with placebo (odds ratio, 2.09; 95\% CI, 1.08 to $4.41 ; \mathrm{P}=0.02$ ) (Fig. S2A in the Supplementary Appendix). In eyes not treated with vitrectomy, the corresponding values were $9.7 \%$ versus $3.7 \%$ (odds ratio, 2.89 95\% CI, 1.26 to $7.76 ; \mathrm{P}=0.008$ ).

Post hoc subgroup analyses stratified by baseline best-corrected visual acuity revealed that at month 6 , a gain of at least three lines was more likely among patients with poorer vision (i.e., baseline best-corrected visual acuity <20/50) than in those with better vision (i.e., baseline best-corrected visual acuity of 20/32 to 20/50 or of $>20 / 32$ ). Among patients with a baseline best-corrected visual acuity that was worse than 20/50, 25.1\% of those treated with ocriplasmin had a gain of three or more lines, as compared with $11.4 \%$ of those given placebo $(\mathrm{P}=0.01)$. Among patients with a baseline best-corrected visual acuity that was better than 20/50, the proportion of eyes that gained three or more lines did not differ significantly between the ocriplasmin and placebo groups (Fig. S2B in the Supplementary Appendix). In all subgroups combined, the mean change, as measured by the number of ETDRS letters gained or lost, was not significant (treatment difference, 1.1; 95\% CI, -0.8 to $2.9 ; \mathrm{P}=0.27$ ).

The mean change in the score on the general quality-of-life VFQ-25 vision subscale was an increase of 6.1 points in the ocriplasmin group and an increase of 2.1 points in the placebo group (difference, 4.0 points; $95 \%$ CI, 1.2 to 6.8; $\mathrm{P}=0.006) .^{22,23}$ The mean change in the overall composite score was an increase of 3.4 points in the ocriplasmin group and an increase of 0.7 points in the placebo group (difference, 2.7 points; $95 \% \mathrm{CI}, 0.7$ to $4.8 ; \mathrm{P}=0.007$ ).

\section{SAFETY}

The proportion of patients who had any ocular adverse event in the study eye was $68.4 \%$ in the ocriplasmin group and $53.5 \%$ in the placebo group $(\mathrm{P}<0.001)$ (Table 2). This difference was driven primarily by adverse events known to be associated with vitreous detachment. Most of the ad-

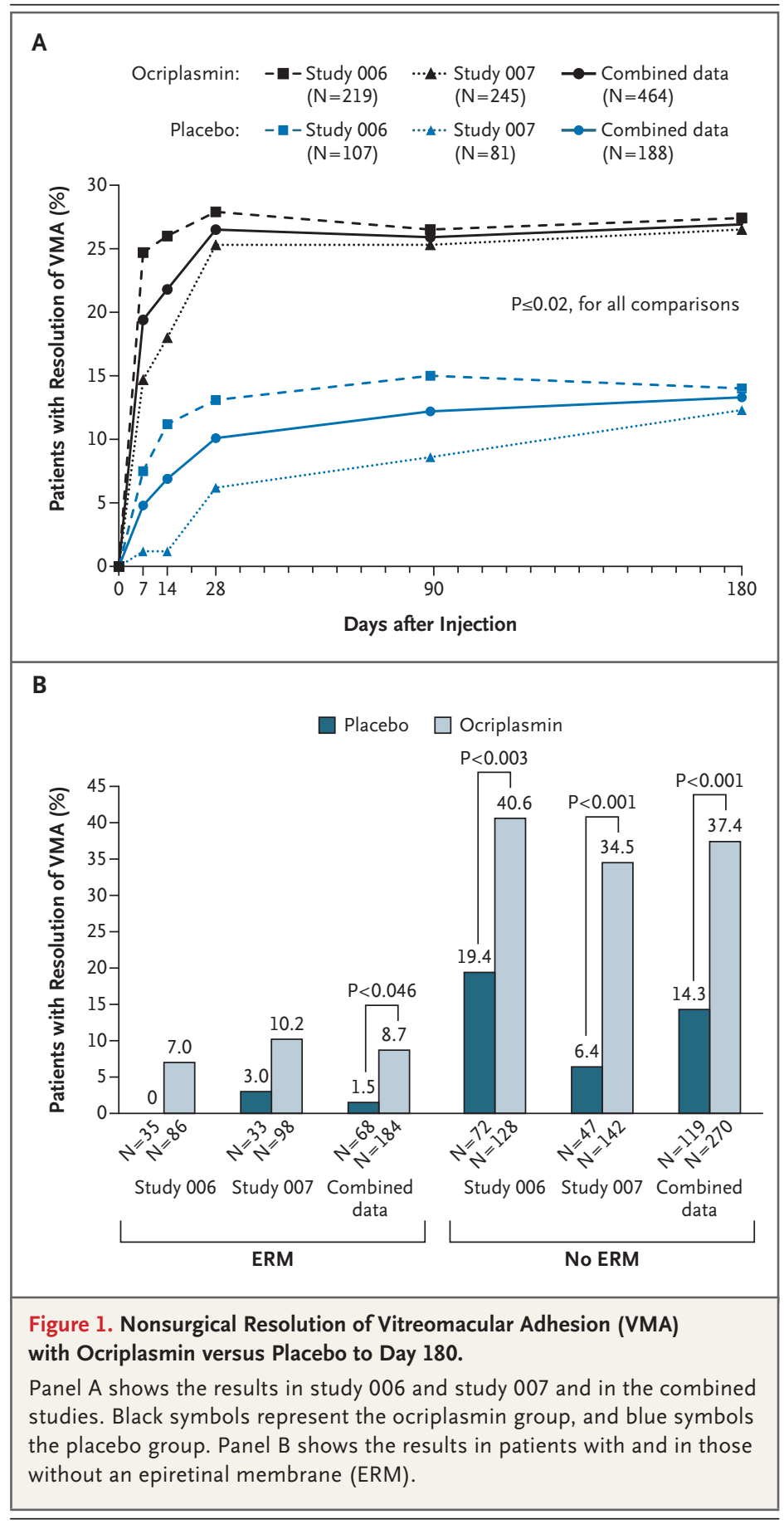

verse events were transient and mild in severity. The most common ocular adverse event in the study eye was vitreous floaters, reported by $16.8 \%$ of patients in the ocriplasmin group and $7.5 \%$ of those in the placebo group (Table 2). The incidence of any serious ocular adverse event in the group treated with ocriplasmin was $7.7 \%$, as 


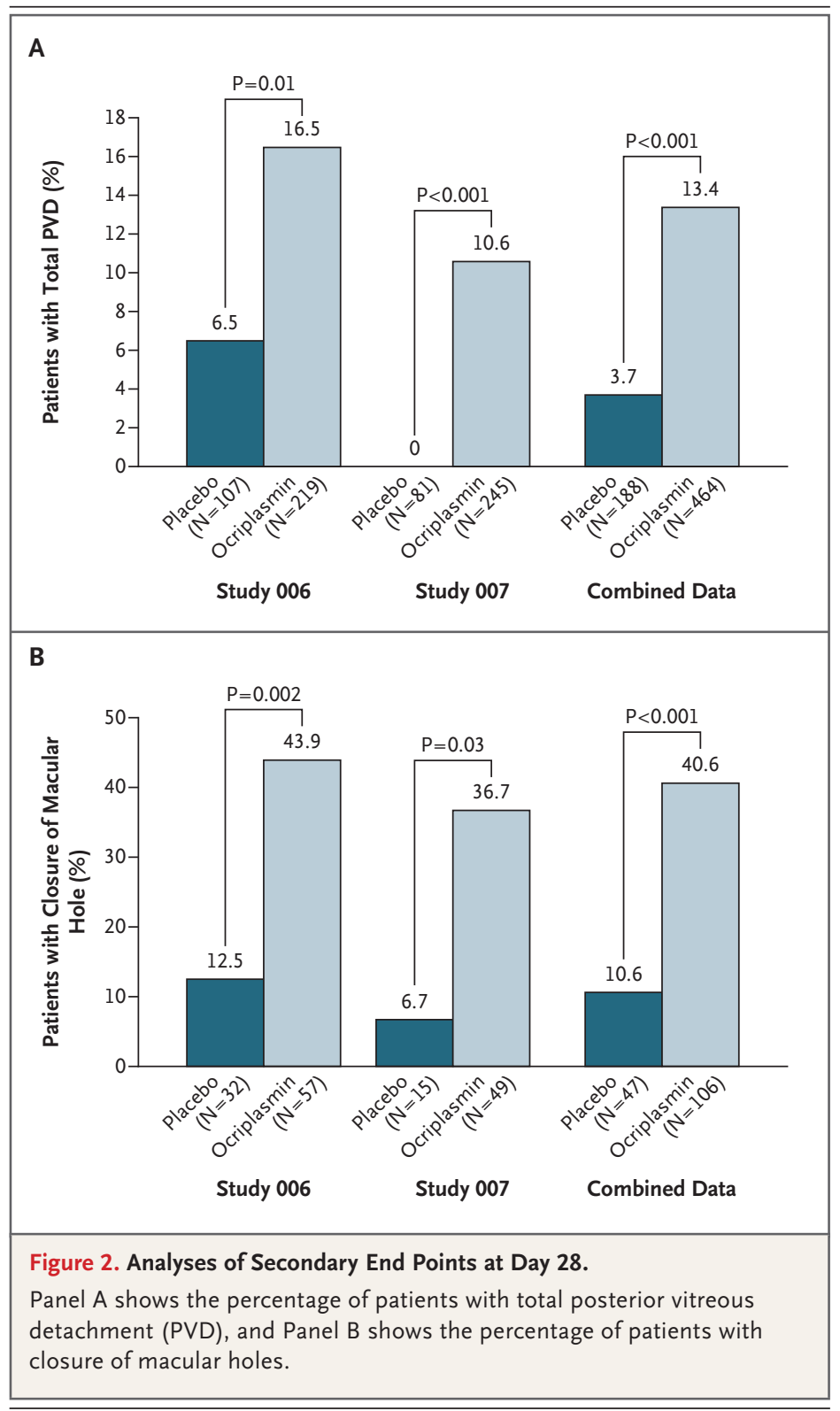

compared with $10.7 \%$ in the placebo group $(\mathrm{P}=0.26)$.

Retinal tears or detachments were diagnosed in $1.9 \%$ of the patients given ocriplasmin, as compared with $4.3 \%$ of those given placebo $(\mathrm{P}=0.11)$. Retinal tears occurred in six of the ocriplasmininjected eyes and in five of the placebo-injected eyes. Most of these tears occurred during vitrectomy, which was performed for vitreomacular traction or macular holes, and were successfully treated intraoperatively. Two retinal tears with detachment occurred before any surgery in the ocriplasmin group and were treated by means of vitrectomy, with successful retinal reattachment. One of these eyes had a best-corrected visual acuity at baseline of 52 ETDRS letters and had recovery to 44 at 6 months; the other eye, with a baseline best-corrected visual acuity of 70 ETDRS letters, had recovery to 62 .

The injections caused no acute cataracts. We observed progression of cataracts in $8.2 \%$ of phakic eyes injected with ocriplasmin and in $11.9 \%$ of phakic eyes injected with placebo $(\mathrm{P}=0.32)$. Among patients who did not undergo vitrectomy, the proportion of patients with cataract progression was similar in the ocriplasmin and placebo groups ( $4.8 \%$ and $5.2 \%$, respectively; $\mathrm{P}=0.97$ ). No cases of endophthalmitis were observed.

\section{DISCUSSION}

Our data support the finding that intravitreal injection of ocriplasmin leads to resolution of vitreomacular traction, induction of posterior vitreous detachment, and closure of a macular hole in some cases. The incidence of vitrectomy was lower among the patients who received ocriplasmin than among those who received placebo. A sham injection would have provided a better comparison with the natural history of the disease process than that provided by the group of patients who received a placebo, which was chosen to control for the effect of an intravitreal injection.

The main visual symptom of vitreomacular traction and macular holes is decreased visual acuity. Although more patients in the ocriplasmin group than in the placebo group had increased visual acuity (defined as a gain of three or more lines of letters) and reported an improved quality of life, the gains were modest. Subgroup analysis suggests that the reason for this finding may be that we included patients with relatively good visual acuity (mean baseline acuity, 64 ETDRS letters; Snellen equivalent, 20/50), creating a ceiling effect whereby only a limited number of patients had sufficiently poor vision to be able to gain three or more lines (Fig. S2B in the Supplementary Appendix). In more general terms, this possibility points to a limitation of the study: the visual acuity of the patients, with the exception of those with macular holes, was better than that of patients for whom vitrectomy would typically be recommended for the treatment of vitreomacular traction and macular holes. 


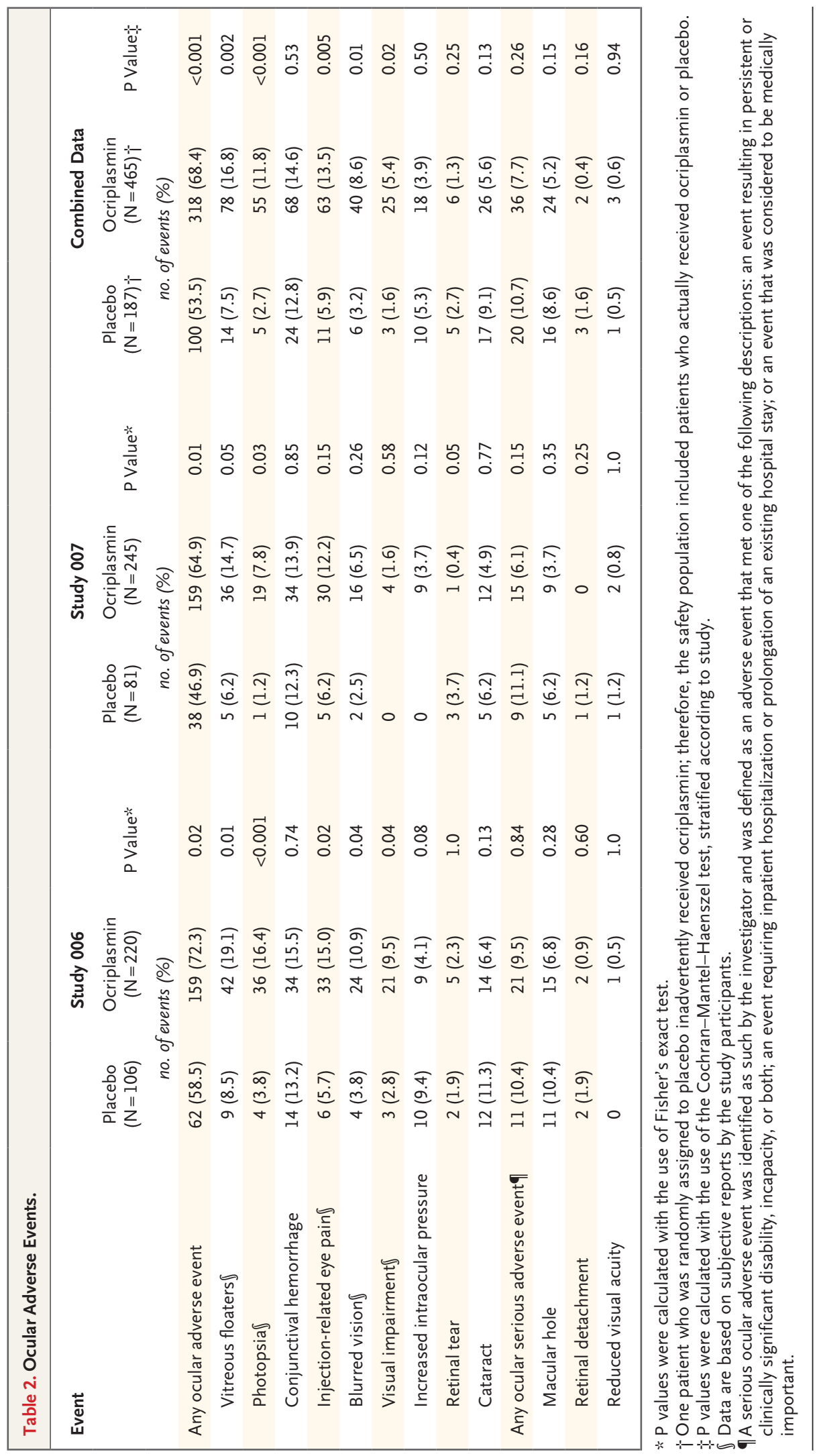

N ENGLJ MED 367;7 NEJM.ORG AUGUST 16, 2012

The New England Journal of Medicine 
We observed increasing lens opacification more often in the placebo group than in the ocriplasmin group, presumably because a higher proportion of eyes in the placebo group underwent vitrectomy. Retinal tears and detachment developed in two eyes after injection of the drug, presumably as a complication of pharmacologically induced posterior vitreous detachment, and these complications were successfully treated with vitrectomy. Overall, more eyes in the placebo group than in the ocriplasmin-treated group had retinal tears and detachment, as reflected by a higher proportion of subjects in the placebo group who required vitrectomy.

Cataract surgery with or without subsequent posterior capsulotomy often induces posterior vitreous detachment soon after the procedure. ${ }^{28,29}$ In this study, any intraocular procedure performed within 3 months before enrollment was an exclusion criterion, reducing the odds that lens status would influence the study outcome. We observed a weak, nonsignificant effect of the intervention on pseudophakic eyes, as compared with its effect on phakic eyes. This may be due to potentially tighter vitreoretinal adhesion in pseudophakic eyes without posterior vitreous detachment, as suggested by the fact that cataract surgery did not detach the vitreous. $^{30}$ The reason for the greater apparent treatment effect in women than in men is also unclear; perhaps it is relevant to factors that make women more susceptible than men to vitreomacular traction, especially macular-hole formation.

Vitreous manipulation effected through intravitreal injection may occasionally result in a posterior vitreous detachment, ${ }^{31,32}$ in which case, the placebo injection of $0.1 \mathrm{ml}$ may have induced some treatment response. The superior therapeutic effects of the ocriplasmin injection would then be indicative of an additional biologic effect of enzymatic vitreolysis over placebo.

The applicability of this study is limited by the exclusion of patients with severe myopia, aphakia, proliferative diabetic retinopathy, or neovascular age-related macular degeneration. ${ }^{9}$ Further studies will be needed to explore the effect of enzymatic vitreolysis in these diseases.

In conclusion, our study shows that enzymatic vitreolysis represents a means to resolve vitreomacular traction and to close macular holes. Intravitreal injection of ocriplasmin was superior to injection of placebo in altering the vitreoretinal interface of affected eyes, although it was accompanied by some, mainly transient, ocular adverse events.

Supported by ThromboGenics, Belgium.

Disclosure forms provided by the authors are available with the full text of this article at NEJM.org.

We thank Chiltern International, the Duke University OCT Reading Center, and Adelphi Communications for their assistance with early drafts of the manuscript and figures and author communications.
REFERENCES

1. Benz MS, Packo KH, Gonzalez V, et al. A placebo-controlled trial of microplasmin intravitreous injection to facilitate posterior vitreous detachment before vitrectomy. Ophthalmology 2010;117:791-7.

2. Ponsioen TL, Hooymans JM, Los LI. Remodelling of the human vitreous and vitreoretinal interface - a dynamic process. Prog Retin Eye Res 2010;29:580-95. 3. Bishop PN. Structural macromolecules and supramolecular organisation of the vitreous gel. Prog Retin Eye Res 2000; 19:323-44.

4. Le Goff MM, Bishop PN. Adult vitreous structure and postnatal changes. Eye (Lond) 2008;22:1214-22.

5. Foos RY, Wheeler NC. Vitreoretinal juncture: synchysis senilis and posterior vitreous detachment. Ophthalmology 1982;89:1502-12.

6. Sebag J. Age-related differences in the human vitreoretinal interface. Arch Ophthalmol 1991;109:966-71.

7. Idem. Anomalous posterior vitreous detachment: a unifying concept in vitreoretinal disease. Graefes Arch Clin Exp Ophthalmol 2004;242:690-8.
8. Gass JD. Stereoscopic atlas of macular diseases: diagnosis and treatment. 4th ed. St. Louis: Mosby, 1997.

9. Johnson MW. Posterior vitreous detachment: evolution and complications of its early stages. Am J Ophthalmol 2010; 149(3):371.e1-382.e1.

10. Krebs I, Brannath W, Glittenberg C, Zeiler F, Sebag J, Binder S. Posterior vitreomacular adhesion: a potential risk factor for exudative age-related macular degeneration? Am J Ophthalmol 2007;144: 741-6.

11. Mojana F, Cheng L, Bartsch DU, et al. The role of abnormal vitreomacular adhesion in age-related macular degeneration: spectral optical coherence tomography and surgical results. Am J Ophthalmol 2008;146:218-27.

12. Hikichi T, Fujio N, Akiba J, Azuma Y, Takahashi M, Yoshida A. Association between the short-term natural history of diabetic macular edema and the vitreomacular relationship in type II diabetes mellitus. Ophthalmology 1997;104:473-8. 13. Lopez-Lopez F, Rodriguez-Blanco $M$, Gómez-Ulla F, Marticorena J. Enzymatic vitreolysis, Curr Diabetes Rev 2009;5:5762.

14. Sebag J. Pharmacologic vitreolysis premise and promise of the first decade. Retina 2009;29:871-4.

15. Rhéaume MA, Vavvas D. Pharmacologic vitreolysis. Semin Ophthalmol 2010; 25:295-302.

16. Verstraeten TC, Chapman C, Hartzer M, Winkler BS, Trese MT, Williams GA. Pharmacologic induction of posterior vitreous detachment in the rabbit. Arch Ophthalmol 1993;111:849-54.

17. de Smet MD, Valmaggia C, ZarranzVentura J, Willekens B. Microplasmin: ex vivo characterization of its activity in porcine vitreous. Invest Ophthalmol Vis Sci 2009;50:814-9.

18. Gandorfer A, Rohleder M, Sethi C, et al. Posterior vitreous detachment induced by microplasmin. Invest Ophthalmol Vis Sci 2004;45:641-7.

19. Gandorfer A. Microplasmin-assisted vitrectomy. Dev Ophthalmol 2009;44:2630.

20. de Smet MD, Gandorfer A, Stalmans $\mathrm{P}$, et al. Microplasmin intravitreal admin- 
istration in patients with vitreomacular traction scheduled for vitrectomy: the MIVI I trial. Ophthalmology 2009;116: 1349-55.

21. Stalmans P, Delaey C, de Smet MD, van Dijkman E, Pakola S. Intravitreal injection of microplasmin for treatment of vitreomacular adhesion: results of a prospective, randomized, sham-controlled phase II trial (the MIVI-IIT trial). Retina 2010;30:1122-7.

22. Mangione CM, Lee PP, Gutierrez PR, Spritzer K, Berry S, Hays RD. Development of the 25-item National Eye Institute Visual Function Questionnaire. Arch Ophthalmol 2001;119:1050-8.

23. Mangione $C M$. The National Eye Institute 25-item Visual Function Questionnaire (VFQ-25) scoring algorithm, version 2000. Bethesda, MD: National Eye Institute, 2000 (http://www.nei.nih.gov/ resources/visionfunction/ manual_cm2000.pdf).
24. DiBernardo CW, Greenberg EF. Ophthalmic ultrasound: a diagnostic atlas. New York: Thieme Medical, 1998.

25. Ehlies F, Chialant D, DiBernardo C. Ultrasound. In: Huang D, Kaiser P, Lowder C, et al., eds. Retinal imaging. Philadelphia: Mosby Elsevier, 2006:81-92. 26. Age-Related Eye Disease Study Research Group. The Age-Related Eye Disease Study (AREDS) system for classifying cataracts from photographs: AREDS report no. 4. Am J Ophthalmol 2001;131:167-75. 27. 27 Orr P, Rentz AM, Margolis MK, et al. Validation of the National Eye Institute Visual Function Questionnaire-25 (NEI VFQ-25) in age-related macular degeneration. Invest Ophthalmol Vis Sci 2011;52: 3354-9.

28. Mirshahi A, Hoehn F, Lorenz K, Hattenbach LO. Incidence of posterior vitreous detachment after cataract surgery. J Cataract Refract Surg 2009;35:987-91.

29. Sheard RM, Goodburn SF, Comer MB， Copyright (؟ 2012 Massachusetts Medical Society.
Scott JD, Snead MP. Posterior vitreous detachment after neodymium:YAG laser posterior capsulotomy. J Cataract Refract Surg 2003;29:930-4.

30. Ivastinovic D, Schwab C, Borkenstein A, Lackner EM, Wedrich A, Velikay-Parel M. Evolution of early changes at the vitreoretinal interface after cataract surgery determined by optical coherence tomography and ultrasonography. Am J Ophthalmol 2012;153:705-9.

31. Rouvas A, Petrou P, Ladas I, Neamonitou G, Vergados I. Spontaneous resolution of vitreomacular traction following ranibizumab (Lucentis) injection. Eur J Ophthalmol 2008;18:301-3.

32. Sivaprasad S, Ockrim Z, Massaoutis P, Ikeji F, Hykin PG, Gregor ZJ. Posterior hyaloid changes following intravitreal triamcinolone and macular laser for diffuse diabetic macular edema. Retina 2008; 28:1435-42.

The Journal requires investigators to register their clinical trials in a public trials registry. The members of the International Committee of Medical Journal Editors (ICMJE) will consider most reports of clinical trials for publication only if the trials have been registered.

Current information on requirements and appropriate registries is available at www.icmje.org/faq_clinical.html. 